\title{
Immunological effects of a low-dose cytarabine, aclarubicin and granulocyte-colony stimulating factor priming regimen on a mouse leukemia model
}

\author{
JINQIU CHEN, NAN YANG, HAILING LIU, HUAN YAO, JIN WANG, YUN YANG and WANGGANG ZHANG \\ Department of Clinical Hematology, Second Affiliated Hospital, \\ Medical School of Xi'an Jiaotong University, Xi'an, Shaanxi 710004, P.R. China
}

Received October 9, 2017; Accepted April 9, 2018

DOI: $10.3892 / \mathrm{ol} .2018 .9018$

\begin{abstract}
The low-dose cytarabine, aclarubicin and granulocyte-colony stimulating factor (G-CSF) (CAG) priming regimen is an effective treatment for patients with relapsed or refractory acute myeloid leukemia (AML) and advanced myelodysplastic syndrome (MDS). G-CSF influences the bone marrow microenvironment (BMM) by mobilizing regulatory T cells (Tregs) and myeloid-derived suppressor cells (MDSCs), as well as by reducing the expression of stromal cell-derived factor- $1 \alpha(\mathrm{SDF}-1 \alpha)$. In the present study, a WEHI-3-grafted BALB/c mouse AML model (AML-M4) was employed to determine how the BMM was altered by different treatment regimens. It was evident that $\mathrm{CAG}$ regimen decreased and increased the proportion of Tregs and MDSCs in the bone marrow and spleen, respectively. Furthermore, the CAG regimen downregulated SDF- $1 \alpha$ levels in the bone marrow and peripheral blood. However, hematoxylin and eosin staining of the main organs revealed that leukemic cells infiltrated the liver following treatment with the $\mathrm{CAG}$ regimen. The present study indicates that the CAG regimen has a positive effect on the immunosuppressive microenvironment in AML and relieves AML-associated BMM immune suppression by decreasing Tregs and MDSCs in the bone marrow and downregulating the $\mathrm{SDF}-1 \alpha / \mathrm{CXCR} 4$ axis in the bone marrow and peripheral blood.
\end{abstract}

\section{Introduction}

Acute myeloid leukemia (AML), which is a clonal disorder of the myeloid line of blood cells, progresses rapidly and

Correspondence to: Professor Wanggang Zhang, Department of Clinical Hematology, Second Affiliated Hospital, Medical School of Xi'an Jiaotong University, 157 West Five Road, Xi'an, Shaanxi 710004, P.R. China

E-mail: zhangwanggang2003@yahoo.com

Key words: acute myeloid leukemia, granulocyte colony-stimulating factor, immune suppression, regulatory $\mathrm{T}$ cells, myeloid-derived suppressor cells, stromal cell-derived factor- $1 \alpha$ is associated with poor clinical prognosis (1). AML most commonly affects older adults, with a median age at diagnosis of 67 years, and 1/3 AML patients are $>75$ years old (1). The ' $3+7$ ' regimen (3 days of anthracycline and 7 days of cytarabine treatment) has remained unchanged for $>30$ years, and has a $60-80 \%$ complete response (CR) rate in adult patients with AML $<60$ years old, and a $40-60 \%$ CR rate in patients $>60$ years old. The overall curative rate of conventional chemotherapy is only $30-40 \%$ in patients $<60$ years old, and $<10 \%$ in elderly patients or those with adverse karyotypes $(2,3)$. Patients who experience relapsed or refractory AML, are associated with poor outcomes (3).

The low-dose cytarabine, aclarubicin and granulocyte-colony stimulating factor (G-CSF) (CAG) priming regimen was first developed by Yamada et al (4) for the treatment of relapsed AML in 1995. CAG chemotherapy has since been investigated as an effective treatment for patients with relapsed or refractory AML and advanced myelodysplastic syndrome (MDS) in Japan and China (5-9). The rationale for this regimen is that G-CSF potentiates the anti-leukemic effect of cytosine arabinoside (AraC) (10-12) by recruiting quiescent G0 leukemic cells into the cell cycle (11), thus improving the CR rate of patients with refractory and relapsed leukemia.

Hematopoietic stem cells (HSCs) exist within the niche of the bone marrow microenvironment (BMM) which regulates stem cell survival, proliferation, differentiation and apoptosis (13). The stromal-derived factor-1 $\alpha$ (SDF-1 $\alpha$ )/C-X-C chemokine receptor type 4 (CXCR4) axis is the key factor associated with HSC chemotaxis $(14-16)$, homing $(17,18)$ and survival/anti-apoptosis $(19,20)$. It has also been demonstrated to dynamically mediate HSC-trafficking in the bone marrow (18). Inhibition of the SDF-1 $\alpha /$ CXCR4 axis using the CXCR4 antagonist, AMD3100, has been indicated to induce leukemia cells to enter the peripheral circulation and induce higher sensitivity to chemotherapeutic drugs (21). G-CSF, an effective stem cell-mobilizing agent, induces SDF- $1 \alpha$ secretion from bone marrow stromal cells into the blood, thus recruiting $\mathrm{CXCR}^{+}$ cells, including HSCs, into the peripheral circulation (22). It has been established that G-CSF can promote the expression of transcriptional repressor growth factor independence-1 (Gfi-1), which binds to DNA sequences upstream of the CXCR4 gene and reduces CXCR4 expression in myeloid cells (23). 
It has been demonstrated that the BMM serves a crucial role in leukemia development and progression, and that it becomes immunosuppressive. Several types of immunosuppressive cells, including regulatory T cells (Tregs) (24), myeloid-derived suppressor cells (MDSCs) (25) and tumor-associated macrophages (TAMs) (26), contribute to the immunologically permissive microenvironment and aid in tumor immune evasion. Tregs and MDSCs mediate their suppressive effects and promote tumor progression by inhibiting T-cell priming $(24,25)$. Furthermore, they provide a favorable microenvironment in which cancer cells can proliferate, expand and evade host immunosurveillance $(24,25)$. As previously reported, SDF-1 $\alpha$ is involved in the chemotaxis and adhesion of Tregs and HSCs $(27,28)$. G-CSF can mobilize Tregs from the bone marrow into the peripheral blood by reducing bone marrow-derived SDF-1 $\alpha$ expression (27). G-CSF has been identified as a major factor in the differentiation of granulocytic MDSCs (gMDSCs), and tumor-derived granulocyte-macrophage colony-stimulating factor (GM-CSF) has been demonstrated to serve a key role in monocytic MDSC (mMDSC) production (29-31).

The present study examined the alteration of the immunosuppressive BMM following administration of G-CSF in combination with low-dose chemotherapy. An established syngeneic leukemia mouse model using the murine AML WEHI-3 cell line $(32,33)$ was used to determine whether the BMM would be altered in vivo following treatment with the CAG priming regimen.

\section{Materials and methods}

Cells and reagents. The murine AML cell line, WEHI-3, was maintained in the laboratory of the Department of Clinical Hematology, Second Affiliated Hospital, Medical School of Xi'an Jiao Tong University (Xi'an, China) in DMEM medium supplemented with $10 \%$ fetal bovine serum(Biological Industries, Israel) and $1 \%$ penicillin-streptomycin $(100 \mathrm{U} / \mathrm{ml}$ penicillin and 100 mg/ml streptomycin; cat no. 15140-122; Gibco; Thermo Fisher Scientific, Inc., Waltham, MA, USA). The following antibodies were used for flow cytometry (FCM): Fluorescein isothiocyanate (FITC) anti-mouse cluster of differentiation (CD)4 (cat no. 11-0042), allophycocyanin (APC) anti-mouse CD25 (cat no. 17-0251), phycoerythrin (PE) anti-mouse/rat Forheac box P3 (Foxp3) (cat no. 12-5773) and PE anti-mouse CD184 (CXCR4; cat no. 12-9991), and all were purchased from eBioscience (Thermo Fisher Scientific, Inc.). FITC anti-mouse CD11b (cat no. 557396), PE anti-mouse lymphocyte antigen 6G (Ly-6G) and Ly-6C (cat no. 561084) were purchased from BD Biosciences (San Jose, CA, USA). The mouse SDF-1 $\alpha$ (cat. no. MCX120), transforming growth factor $\beta$ (TGF- $\beta$; cat. no. MB100B) and interleukin 10 (IL-10; cat. no. M1000B) ELISA kits were purchased from R\&D Systems, Inc. (Minneapolis, MN, USA), and the arginase 1 (Arg-1; cat. no. JL13668) ELISA kit was purchased from and Shanghai Jiang Lai Biotechnology Co., Ltd. (Shanghai, China; http://www.laibio.com/).

Leukemia mouse model and chemotherapy regimens. All animal experiments were reviewed and approved by the Ethics Committee of the Medical College, Xi'an Jiao Tong University (Xi'an, China). Male BALB/c wild-type mice (6-8 weeks old, weighed $21 \pm 2 \mathrm{~g}$ ) were purchased from the Laboratory Animal
Center, Medical College of Xi'an Jiao Tong University. The mice were maintained under specific pathogen-free conditions, in a $12 / 12 \mathrm{~h}$ light/dark cycle at $21 \pm 2^{\circ} \mathrm{C}$, with access to food and water ad libitum. A total of 21 mice were randomly divided into 3 groups of 7 . For the syngeneic acute myelomonocytic leukemia model (AML-M4), BALB/c mice were injected intravenously into the tail vein on day 0 with $1 \times 10^{6}$ WEHI-3 cells in $100 \mu \mathrm{l}$ PBS as previously described (32-34). On day 15, all 7 mice in the AML (control) group were humanely sacrificed and the spleen, liver, femur and blood were collected and stored for further experiments. For the daunorubicin and cytarabine (DNR-AraC; DA) group, 7 mice were treated with $10 \mathrm{mg} / \mathrm{kg}$ DNR intravenously on days 15-17 and $50 \mathrm{mg} / \mathrm{kg}$ AraC intraperitoneally on days 15-21. Mice in DA group were sacrificed on day 22 and the spleen, liver, femur and blood were collected for further experiments. For the CAG group, 7 mice were treated with $6.25 \mathrm{mg} / \mathrm{kg}$ AraC via hypodermic injection (IH) on days $15-18,3 \mathrm{mg} / \mathrm{kg}$ aclarubicin (ACR) intravenously on days $15-28$ and $0.2 \mathrm{mg} / \mathrm{kg}$ G-CSF via IH on days 15-28. All mice in CAG group were sacrificed on day 29 and the spleen, liver, femur and blood were collected for further experiments.

ELISA. SDF-1 $\alpha$, IL-10 and Arg-1 levels in the blood plasma were determined using the aforementioned commercially available mouse ELISA kits, according the manufacturers' protocols. Absorbance was measured at $450 \mathrm{~nm}$ on a microplate reader (Thermo Fisher Scientific, Inc.).

FCM analysis. Single-cell suspensions of spleen cells and bone marrow mononuclear cells (BMMCs) were stained with FITC-conjugated anti-mouse CD4 (dilution, 1:100) and APC-conjugated anti-mouse CD25 (dilution, 1:100) for $30 \mathrm{~min}$ on ice. The cells were washed with flow cytometry staining buffer (eBioscience; Thermo Fisher Scientific, Inc.), resuspended in $1 \mathrm{ml}$ fixation/permeabilization working solution (eBioscience; Thermo Fisher Scientific, Inc.) and incubated at $4^{\circ} \mathrm{C}$, in the dark, overnight. The cells were washed twice with $1 \mathrm{X}$ permeabilization buffer (eBioscience; Thermo Fisher Scientific, Inc.) and incubated for $30 \mathrm{~min}$ at $4^{\circ} \mathrm{C}$ in $1 \mathrm{X}$ permeabilization buffer containing $\mathrm{PE}$-conjugated anti-mouse Foxp3 (dilution, 1:40) antibody. Forward vs. side scatter was plotted for lymphocytes, followed by gating for $\mathrm{CD}^{+} \mathrm{T}$ cells. These cells were then analyzed for the expression of CD25 and FoxP3. For the detection of MDSCs and CXCR4, single-cell suspensions were stained with CD11b (dilution, 1:200), Gr-1 (Ly-6G and Ly-6C; dilution, 1:200) or CXCR4 (dilution, 1:20) antibodies. FCM was performed using a FACS flow cytometer (BD Biosciences, Franklin Lakes, NJ, USA) and the data was analyzed using CellQuest Pro (version 6.0; BD Biosciences).

Pathological examinations. The femur, liver and spleen tissues were harvested and immediately fixed in $10 \%$ neutral-buffered formalin for $24 \mathrm{~h}$. Liver and spleen tissues were embedded in paraffin and the femurs were decalcified in 10\% EDTA for $\sim 30$ days at room temperature prior to being embedded in paraffin. A total of 54- $\mu$ m-thick sections were cut from each paraffin block. The sections were stained at room $25^{\circ} \mathrm{C}$ using hematoxylin $(\mathrm{H})$ for $6 \mathrm{~min}$ and eosin $(\mathrm{E})$ for $1 \mathrm{~min}$ (cat no. C0105; Beyotime Institute of Biotechnology, Shanghai, China), 
A

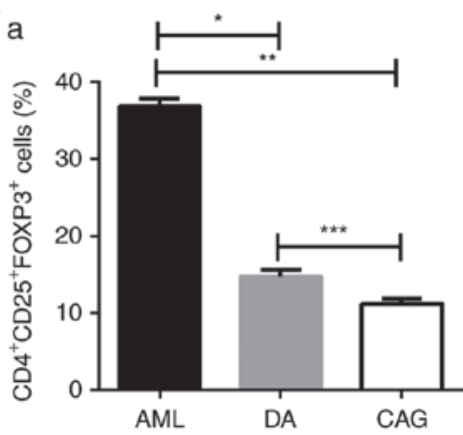

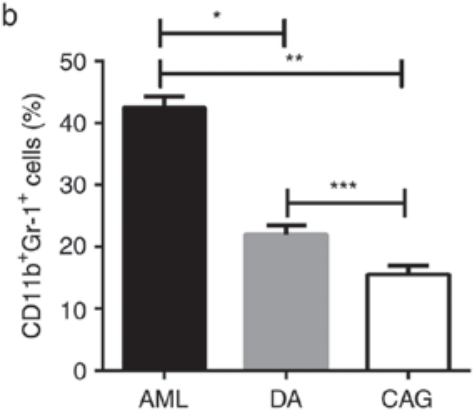

B a

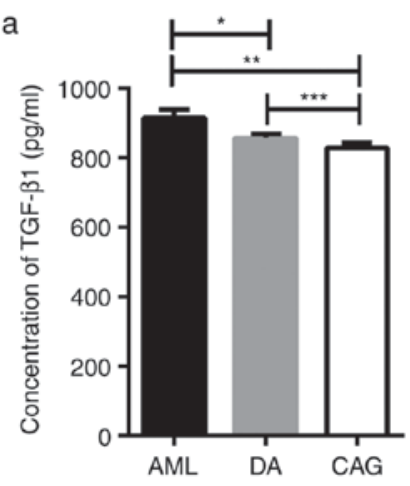

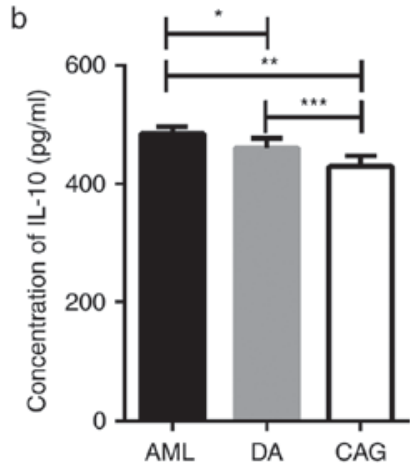

C

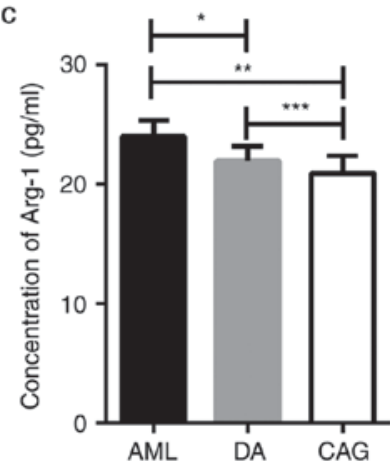

Figure 1. Tregs and MDSC numbers and cytokines levels in the bone marrow are reduced with the CAG regimen. $(\mathrm{A})(\mathrm{a}) \mathrm{Percentage}$ of $\mathrm{CD} 4^{+} \mathrm{CD} 25^{+} \mathrm{Foxp} 3^{+}$ Tregs in each group, analyzed by FCM. ${ }^{*} \mathrm{P}<0.01,{ }^{* *} \mathrm{P}<0.01,{ }^{* * *} \mathrm{P}<0.05$; (b) Percentage of CD11b ${ }^{+} \mathrm{Gr}-1^{+} \mathrm{MDSC}$ in each group, analyzed by FCM. ${ }^{*} \mathrm{P}<0.01$, ${ }^{* *} \mathrm{P}<0.01,{ }^{* * *} \mathrm{P}<0.05$. (B) (a) Concentration of TGF- $\beta 1$ in the bone marrow supernatant, analyzed by ELISA. ${ }^{*} \mathrm{P}<0.01,{ }^{* *} \mathrm{P}<0.01,{ }^{* * *} \mathrm{P}<0.01 ;(\mathrm{b}) \mathrm{Concentration}$ of IL-10 in bone marrow supernatant, analyzed by ELISA. ${ }^{*} \mathrm{P}<0.01,{ }^{* *} \mathrm{P}<0.01,{ }^{* * *} \mathrm{P}<0.01$; (c) Concentration of Arg-1 in bone marrow supernatant, analyzed by ELISA. ${ }^{*} \mathrm{P}<0.01,{ }^{* *} \mathrm{P}<0.01,{ }^{* * * *} \mathrm{P}<0.05$. Tregs, regulatory T cells; MDSCs, myeloid-derived suppressor cells; CAG, cytarabine + aclarubicin + granulocyte-colony stimulating factor; $\mathrm{CD}$, cluster of differentiation; Foxp3, forkhead box protein 3 ; FCM, flow cytometry; TGF- $\beta 1$, transforming growth factor- $\beta 1$; IL- 10 , interleukin-10; Arg-1, arginase-1; AML, acute myeloid leukemia; DA, daunorubicin + cytarabine.

according to standard histochemical procedures. Images were acquired using a light microscope (DP71; Olympus Corporation, Tokyo, Japan).

Statistical analysis. All data are expressed as the mean \pm standard deviation. Differences between treatment groups were determined using analysis of variance followed by Fisher's Least Significant Difference post-hoc test. $\mathrm{P}<0.05$ was considered to indicate a statistically significant difference. All analyses were performed using SPSS 18.0 software (SPSS Inc., Chicago, IL, USA).

\section{Results}

$C A G$ treatment regimen decreases the number of Tregs and MDSCs in bone marrow. To determine whether the CAG and DA treatment regimens affect AML immunosuppressive cells in the BMM, FCM was performed to investigate the percentage of $\mathrm{CD}^{+}{ }^{+} \mathrm{CD} 25^{+} \mathrm{FoxP} 3^{+}$Tregs and $\mathrm{CD} 11 \mathrm{~b}^{+} \mathrm{Gr}-1^{+}$ MDSCs in BMMCs. It was revealed that the levels of Tregs and MDSCs in the DA and CAG groups were significantly lower than in the AML group $(\mathrm{P}<0.01$; Fig. 1A). Furthermore, compared with mice in the DA group, mice in CAG group exhibited significantly decreased levels of Tregs and MDSCs in BMMCs $(\mathrm{P}<0.05$; Fig. 1A).

CAG treatment regimen decreases Tregs and MDSCs associated cytokine levels in the BMM. TGF- $\beta$ and IL-10 are anti-inflammatory and immunosuppressive cytokines secreted by Tregs $(35,36)$, while Arg-1, which suppresses T-lymphocyte function, is expressed by MDSCs $(37,38)$. The ELISA results demonstrated that the concentrations of TGF- $\beta$, IL-10 and Arg-1 in the bone marrow supernatant were significantly decreased in the DA $(\mathrm{P}<0.01)$ and CAG $(\mathrm{P}<0.01)$ groups compared with the AML group (Fig. 1B). The CAG group exhibited lower TGF- $\beta 1(\mathrm{P}<0.01)$, IL-10 $(\mathrm{P}<0.01)$ and Arg-1 $(\mathrm{P}<0.05)$ concentrations compared with the DA group (Fig. 1B). These results suggest that the CAG treatment regimen may rescue immune suppression in AML bone marrow.

Compared with the DA treatment regimen, the CAG treatment regimen increases Treg and MDSC numbers in the spleen. The percentages of Tregs and MDSCs in mice spleen single cell suspensions were tested by FCM. As shown in Fig. 2, mice in the DA and CAG groups both exhibited lower percentages of Tregs and MDSCs in spleen than AML group $(\mathrm{P}<0.01)$. However, there were significantly higher levels of Tregs $(\mathrm{P}<0.01)$ and MDSCs $(\mathrm{P}<0.05)$ in the CAG group than the DA group.

$C A G$ treatment regimen downregulates $S D F-1 \alpha$ levels in the bone marrow. The level of SDF-1 $\alpha$ in bone marrow supernatant was detected by ELISA, and the results revealed that the concentration of SDF- $1 \alpha$ in the CAG group was significantly lower than the AML and DA groups $(\mathrm{P}<0.01)$. However, there was no significant difference (in the concentration of bone marrow SDF-1 $\alpha$ between AML and DA groups ( $\mathrm{P}>0.05$; Fig. 3 ). 

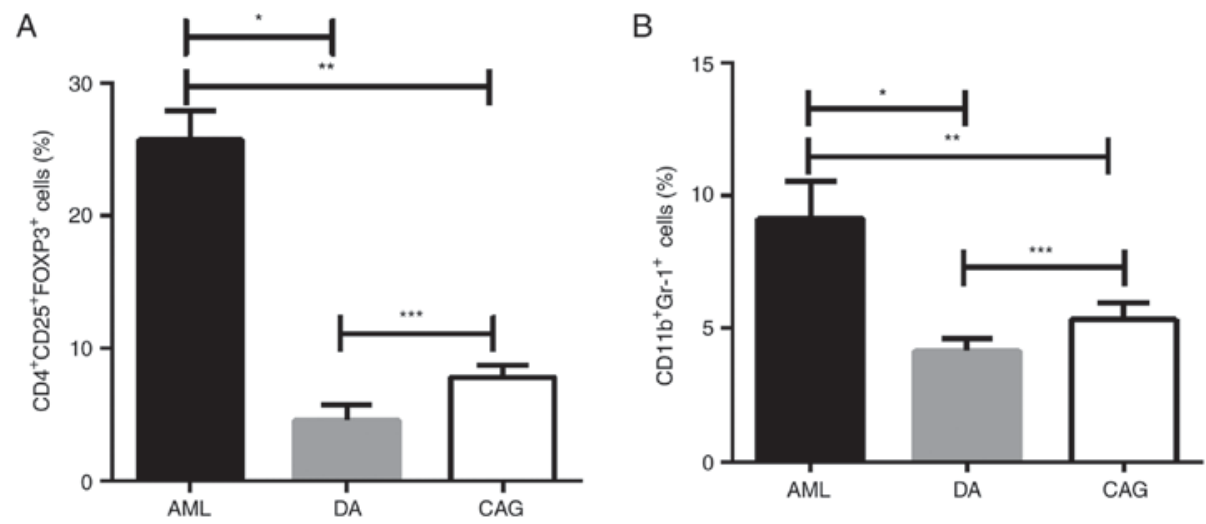

Figure 2. CAG regimen increases Tregs and MDSC numbers in the spleen compared with the DA regimen. (A) Percentage of $\mathrm{CD} 4{ }^{+} \mathrm{CD} 25^{+} \mathrm{FoxP} 3^{+} \mathrm{Tregs}$ in each group, analyzed by FCM. ${ }^{*} \mathrm{P}<0.01,{ }^{* * *} \mathrm{P}<0.01,{ }^{* * * *} \mathrm{P}<0.01$. (B) Percentage of $\mathrm{CD} 11 \mathrm{~b}^{+} \mathrm{Gr}-1^{+}$MDSCs in each group, analyzed by FCM. ${ }^{*} \mathrm{P}<0.01,{ }^{* *} \mathrm{P}<0.01$, ${ }^{* * * *} \mathrm{P}<0.05$. CAG, cytarabine + aclarubicin + granulocyte-colony stimulating factor; Tregs, regulatory T cells; MDSCs, myeloid-derived suppressor cells; DA, daunorubicin + cytarabine; CD, cluster of differentiation; Foxp3, forkhead box protein 3; FCM, flow cytometry; AML, acute myeloid leukemia.

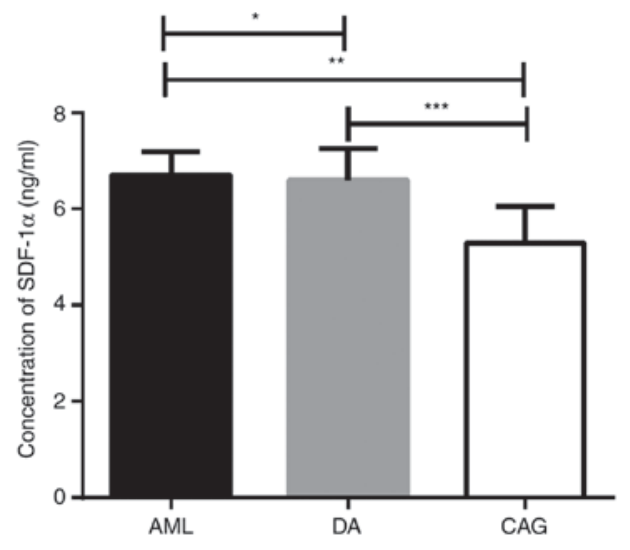

Figure 3. CAG regimen downregulates SDF-1 $\alpha$ levels in the bone marrow. The concentration of SDF-1 $\alpha$ in the bone marrow supernatant was analyzed by ELISA. ${ }^{*} \mathrm{P}>0.05,{ }^{* *} \mathrm{P}<0.01,{ }^{* * *} \mathrm{P}<0.01$. CAG, cytarabine + aclarubicin + granulocyte-colony stimulating factor; SDF-1 $\alpha$, stromal-derived factor-1 $\alpha$; AML, acute myeloid leukemia; DA, daunorubicin + cytarabine.

$C A G$ treatment regimen reduces $S D F-1 \alpha$ levels in the peripheral blood. ELISA results demonstrated that the CAG group exhibited the lowest levels of SDF-1 $\alpha$ in the peripheral blood among the 3 groups of mice (Fig. 4). In consistence with the concentration of SDF-1 $\alpha$ in the bone marrow, there was no significant difference in the concentration of SDF-1 $\alpha$ in the peripheral blood between the AML and DA groups $(\mathrm{P}>0.05)$.

Histopathology of the femur, liver and spleen tissues. Macroscopically, the femurs of AML mice were pale (Fig. 5Aa) and the liver and spleen were swollen (Fig. 5Ab and c) compared with the DA and CAG mice. The DA (Fig. 5Ba) and CAG (Fig. 5Ca) treatment regimens reddened the femurs and decreased the size of the liver and spleen (Fig. 5Bb and c; Fig. 5Cb and c). Leukemic cells in the bone marrow cavity of mice from the AML group were visible under the light microscope (Fig. 6Aa). By contrast, the femur sections from mice of the DA and CAG groups exhibited a recovered hematopoietic system (Fig. 6Ba and Ca). In AML mice, leukemic cells infiltrated the liver and spleen (Fig. 6Ab and c). However, in the DA group, fewer leukemic cells

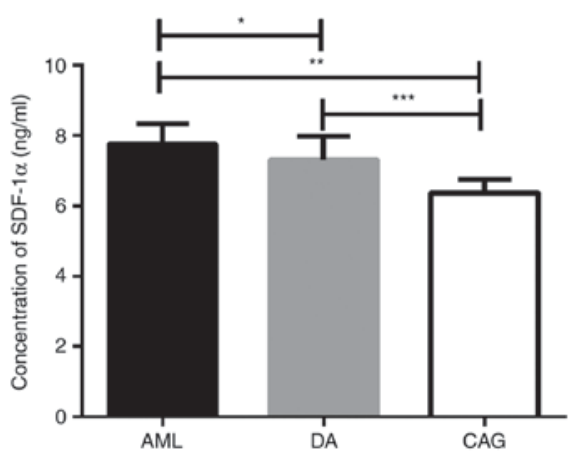

Figure 4. CAG regimen reduces SDF-1 $\alpha$ levels in the peripheral blood. The concentration of SDF-1 $\alpha$ in the peripheral blood in each group, analyzed by ELISA. ${ }^{*} \mathrm{P}>0.05,{ }^{* *} \mathrm{P}<0.01,{ }^{* * *} \mathrm{P}<0.01$. CAG, cytarabine + aclarubicin + granulocyte-colony stimulating factor; SDF- $1 \alpha$, stromal-derived factor- $1 \alpha$. AML, acute myeloid leukemia; DA, daunorubicin + cytarabine.

infiltrated the liver and spleen and the pathological sections exhibited liver fibrosis and spleen trabecula fibrosis (Fig. 6Bb and c). In the CAG group, leukemic cells infiltrated the hepatic portal and central veins (Fig. 6Cb) indicated by the arrows. In the spleens, the boundary of red pulp widened and the white pulp was clear (Fig. 6Cc).

\section{Discussion}

G-CSF has been widely used in the treatment of refractory and relapsed AML together with chemotherapeutic drugs, and has improved the CR rate (13). Although it is well-established that G-CSF has an important influence on the BMM (22), there is little research regarding the effect of G-CSF priming chemotherapy on the BMM. G-CSF can promote leukemia cells to be released from the bone marrow into the peripheral circulation by blocking the interaction of SDF- $1 \alpha$ and CXCR4, thus enhancing the anti-leukemia effect (13). G-CSF also mobilizes immunosuppressive Tregs from the bone marrow into the peripheral blood (27). We hypothesize that there is an effect of the G-CSF priming regimen on the BMM in AML.

An AML-M4 BALB/c mouse model was established to study the BMM in vivo in mice receiving different chemotherapy regimens. Changes in the SDF-1 $\alpha / C X C R 4$ axis, 

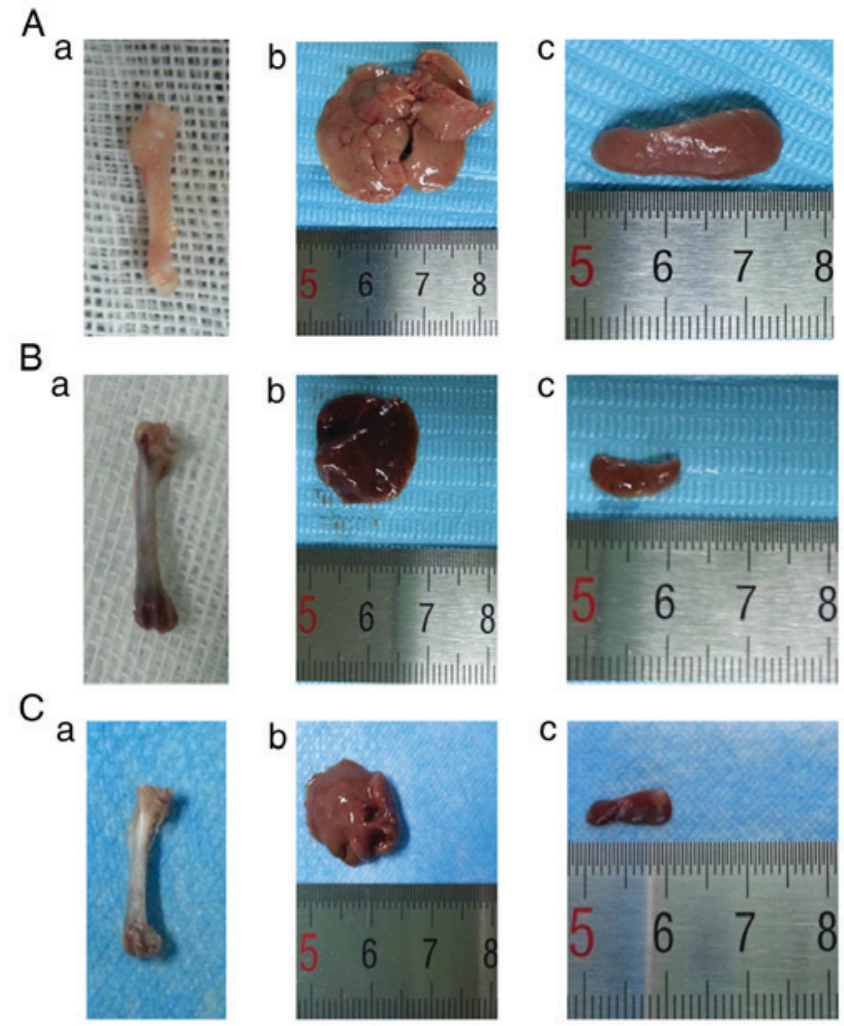

Figure 5. Macroscopic pathology of the femur, liver and spleen. (A) AML group: (a) femur; (b) liver, and (c) spleen. (B) DA group: (a) femur; (b) liver, and (c) spleen. (C) CAG group: (a) femur; (b) liver, and (c) spleen. AML, acute myeloid leukemia; DA, daunorubicin + cytarabine; CAG, cytarabine + aclarubicin + granulocyte-colony stimulating factor.
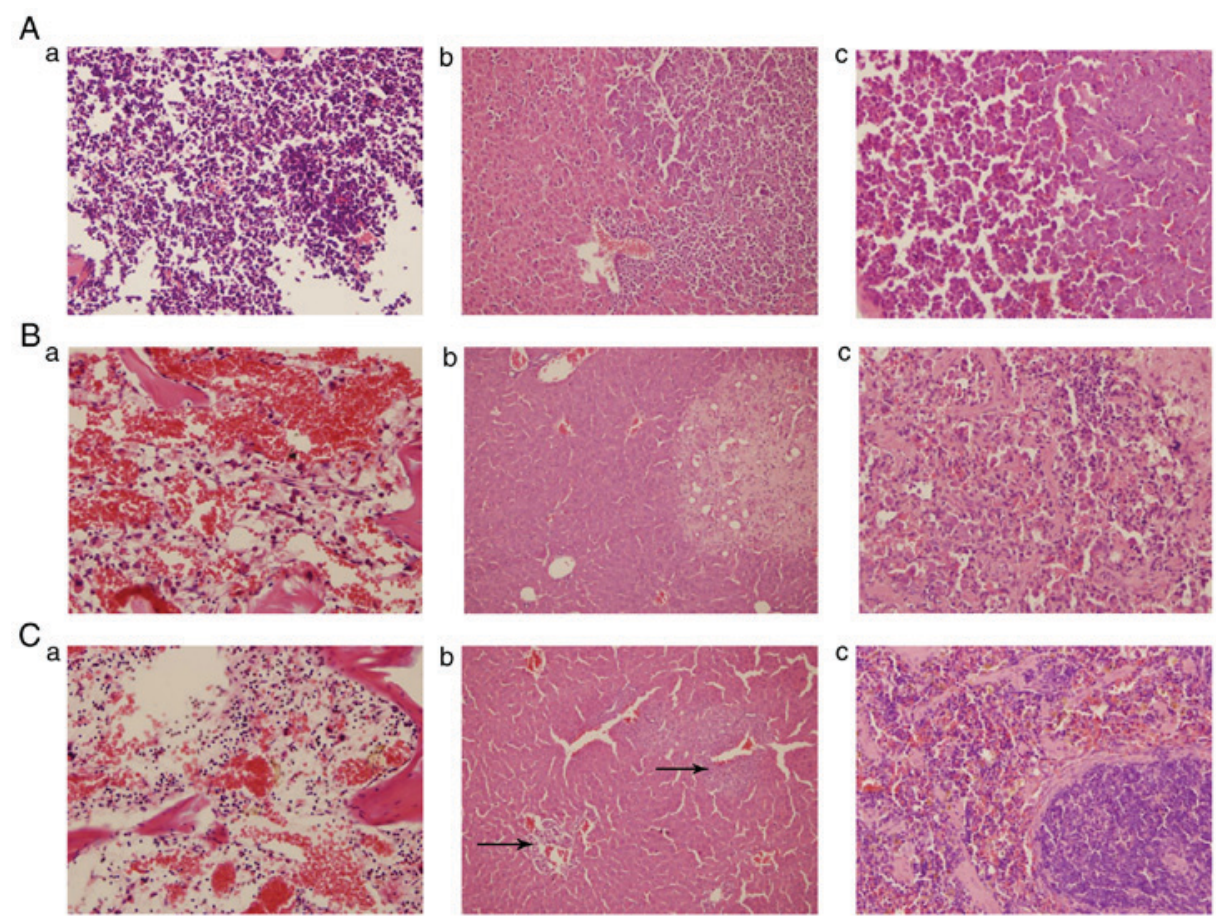

Figure 6. H\&E staining of the femur, liver and spleen. (A) AML group: (a) femur, magnification, x400; (b) liver, magnification, x200; (c) spleen, magnification, x400. (B) DA group: (a) femur, magnification, x400; (b) liver, magnification, x200; (c) spleen, magnification, x400. (C) CAG group: (a) femur, magnification, x400; (b) liver, arrows indicate the infiltration of leukemic cells into the hepatic portal and central veins; magnification, x200; (c) spleen, magnification, x400. H\&E, hematoxylin and eosin; AML, acute myeloid leukemia; DA, daunorubicin + cytarabine; CAG, cytarabine + aclarubicin + granulocyte-colony stimulating factor.

Tregs, MDSCs and associated cytokines were investigated. In accordance with previous studies, it was demonstrated that the
CAG regimen had a positive effect on the immunosuppressive microenvironment in AML. 
In the AML-M4 BALB/c mouse model, the number of Tregs and MDSCs in the bone marrow decreased following CAG chemotherapy. The concentrations of immunosuppressive cytokines (TGF- $\beta 1$, IL-10 and Arg-1), secreted by Tregs and MDSCs, were lower in the CAG group compared with the DA group. The number of Tregs and MDSCs in the spleen, the largest immune organ, was high. This suggests that G-CSF may mobilize Tregs and MDSCs from the bone marrow to the periphery, and that the G-CSF priming regimen may relieve AML-associated BMM immune suppression by reducing the number of MDSCs and Tregs in the bone marrow. This may help to explain how G-CSF administration reduces the severity of acute graft-vs.-host disease (GVHD) (27).

The BMM provides a protective niche for HSCs (28) and it is the primary site of minimal residual disease (MRD) following chemotherapy (39). By hijacking the HSC niche (40), leukemia stem cells (LSCs) in the bone marrow niche serve roles in leukemia initiation, maintenance and recurrence (41). G-CSF priming chemotherapy treatment significantly increased LSC apoptosis and increases responsiveness to chemotherapy in vivo by modifying the functional behavior of LSCs (42). On the other hand, G-CSF inhibits the interaction of SDF- $1 \alpha$ and CXCR4, thus reducing LSC migration to the BMM, which involves CXCR4 (43). In the present study, it was also demonstrated that mice in the CAG group expressed the lowest level of SDF-1 $\alpha$ in the bone marrow and peripheral blood, which indicates that the SDF-1 $\alpha / \mathrm{CXCR} 4$ axis may have been downregulated in the CAG treatment regimen.

However, the CAG treatment regimen is limited in that there were more residual leukemic cells subsequent to treatment in the livers of the CAG mice than the DA mice. This may result from the low dose of the chemotherapeutic agents, AraC and ACR. However, the low doses used in the CAG regimen result in less severe clinical side-effects than common induction chemotherapy, such as the DA regimen. Due to its milder toxicity and a short duration of G-CSF neutropenia treatment, the $\mathrm{CAG}$ regimen is suitable for elderly patients $(5,44,45)$.

The present study suggests that the CAG leukemia treatment regimen has a positive effect on the immunosuppressive microenvironment in AML and relieves AML-associated BMM immune suppression by decreasing the number of Tregs and MDSCs in the bone marrow. The results indicate that downregulation of the SDF-1 $1 \alpha / \mathrm{CXCR} 4$ axis in the bone marrow and peripheral blood is caused by CAG chemotherapy. The lower dose of chemotherapeutic agents in the CAG regimen leads to milder side-effects but more residual leukemic cells. Overall, the CAG regimen relieves immune suppression in the leukemic BMM and is suitable for elderly patients.

\section{Acknowledgments}

Not applicable.

\section{Funding}

The present study was supported by the National Natural Science Foundation of China (grant no. 81270597).

\section{Availability of data and materials}

The datasets used and/or analyzed during the current study are available from the corresponding author on reasonable request.

\section{Authors' contributions}

JC performed the murine model and chemotherapy regimens, performed the majority of the experiments and was a major contributor in writing the manuscript. NY performed FCM, analyzed the FCM data and helped in writing the manuscript. HL and HY sacrificed all the mice and harvested the organs and pretreated them. JW performed pathological examinations and acquired data of these experiments. YY performed ELISA and acquired data of this experiment. WZ designed the study, revised the manuscript and gave final approval of the version to be published. All authors read and approved the final manuscript

\section{Ethics approval and consent to participate}

All animal experiments were reviewed and approved by the Ethics Committee of the Medical College, Xi'an Jiaotong University (Xi'an, China).

\section{Patient consent for publication}

Not applicable.

\section{Competing interests}

The authors declare that they have no competing interests.

\section{References}

1. Podoltsev NA, Stahl M, Zeidan AM and Gore SD: Selecting initial treatment of acute myeloid leukaemia in older adults. Blood Rev 31: 43-62, 2017.

2. Döhner H, Weisdorf DJ and Bloomfield CD: Acute myeloid leukemia. N Engl J Med 373: 1136-1152, 2015.

3. Döhner H, Estey EH, Amadori S, Appelbaum FR, Büchner T, Burnett AK, Dombret H, Fenaux P, Grimwade D, Larson RA, et al: Diagnosis and management of acute myeloid leukemia in adults: Recommendations from an international expert panel, on behalf of the European LeukemiaNet. Blood 115: 453-474, 2010

4. Yamada K, Furusawa S, Saito K, Waga K, Koike T, Arimura H, Aoyagi A, Yamato H, Sakuma H, Tsunogake S, et al: Concurrent use of granulocyte colony-stimulating factor with low-dose cytosine arabinoside and aclarubicin for previously treated acute myelogenous leukemia: A pilot study. Leukemia 9: 10-14, 1995.

5. Minakata D, Fujiwara S, Ito S, Mashima K, Umino K, Nakano H, Kawasaki Y, Sugimoto M, Yamasaki R, Yamamoto C, et al: A low-dose cytarabine, aclarubicin and granulocyte colony-stimulating factor priming regimen versus a daunorubicin plus cytarabine regimen as induction therapy for older patients with acute myeloid leukemia: A propensity score analysis. Leuk Res 42: 82-87, 2016.

6. Jin J, Chen J, Suo S, Qian W, Meng H, Mai W, Tong H, Huang J, $\mathrm{Yu}$ W, Wei J and Lou Y: Low-dose cytarabine, aclarubicin and granulocyte colony-stimulating factor priming regimen versus idarubicin plus cytarabine regimen as induction therapy for older patients with acute myeloid leukemia. Leuk Lymphoma 56: 1691-1697, 2015.

7. Wang Y, Li W, Chen S, Qiu H, Sun A and Wu D: Salvage chemotherapy with low-dose cytarabine and aclarubicin in combination with granulocyte colony-stimulating factor priming in patients with refractory or relapsed acute myeloid leukemia with translocation (8;21). Leuk Res 35: 604-607, 2011. 
8. Liu L, Qu Q, Jiao W, Zhang Y, Li X, Ding C and Wu D: Increasing aclarubicin dose in low-dose cytarabine and aclarubicin in combination with granulocyte colony-stimulating factor (CAG regimen) is efficacious as salvage chemotherapy for relapsed/refractory mixed-phenotype acute leukemia. Leuk Res 39: 805-811, 2015.

9. Ma X, Wang J, Xu Y, Zhang W, Liu J, Cao X, He A, Wang F, Gu L, Lei B and Wang J: Dose-enhanced combined priming regimens for refractory acute myeloid leukemia and middle-and-high-risk myelodysplastic syndrome: A single-center, retrospective cohort study. Onco Targets Ther 9: 3661-3669, 2016.

10. Miyauchi J, Kelleher CA, Wang C, Minkin S and McCulloch EA Growth factors influence the sensitivity of leukemic stem cells to cytosine arabinoside in culture. Blood 73: 1272-1278, 1989.

11. Tafuri A and Andreeff M: Kinetic rationale for cytokine-induced recruitment of myeloblastic leukemia followed by cycle-specific chemotherapy in vitro. Leukemia 4: 826-834, 1990.

12. te Boekhorst PA, Löwenberg B, Vlastuin M and Sonneveld P: Enhanced chemosensitivity of clonogenic blasts from patients with acute myeloid leukemia by G-CSF, IL-3 or GM-CSF stimulation. Leukemia 7: 1191-1198, 1993.

13. Shen ZH, Zeng DF, Ma YY, Zhang X, Zhang C and Kong PY: Are there any new insights for G-CSF and/or AMD3100 in chemotherapy of haematological malignants? Med Oncol 32: 262, 2015.

14. Kim CH and Broxmeyer HE: In vitro behavior of hematopoietic progenitor cells under the influence of chemoattractants: Stromal cell-derived factor-1, steel factor, and the bone marrow environment. Blood 91: 100-110, 1998.

15. Jo DY, Rafii S, Hamada T and Moore MA: Chemotaxis of primitive hematopoietic cells in response to stromal cell-derived factor-1. J Clin Invest 105: 101-111, 2000.

16. Wright DE, Bowman EP, Wagers AJ, Butcher EC and Weissman IL: Hematopoietic stem cells are uniquely selective in their migratory response to chemokines. J Exp Med 195: 1145-1154, 2002.

17. Peled A, Petit I, Kollet O, Magid M, Ponomaryov T, Byk T, Nagler A, Ben-Hur H, Many A, Shultz L, et al: Dependence of human stem cell engraftment and repopulation of NOD/SCID mice on CXCR4. Science 283: 845-848, 1999.

18. Lapidot $\mathrm{T}$ and Kollet $\mathrm{O}$ : The essential roles of the chemokine SDF-1 and its receptor CXCR4 in human stem cell homing and repopulation of transplanted immune-deficient NOD/SCID and NOD/SCID/B2m(null) mice. Leukemia 16: 1992-2003, 2002

19. Broxmeyer HE, Cooper S, Kohli L, Hangoc G, Lee Y, Mantel C, Clapp DW and Kim CH: Transgenic expression of stromal cell-derived factor-1/CXC chemokine ligand 12 enhances myeloid progenitor cell survival/antiapoptosis in vitro in response to growth factor withdrawal and enhances myelopoiesis in vivo. J Immunol 170: 421-429, 2003.

20. Broxmeyer HE, Kohli L, Kim CH, Lee Y, Mantel C, Cooper S, Hangoc G, Shaheen M, Li X and Clapp DW: Stromal cell-derived factor-1/CXCL12 directly enhances survival/antiapoptosis of myeloid progenitor cells through CXCR4 and G(alpha)i proteins and enhances engraftment of competitive, repopulating stem cells. J Leukoc Biol 73: 630-638, 2003.

21. Zeng Z, Shi YX, Samudio IJ, Wang RY, Ling X, Frolova O, Levis M, Rubin JB, Negrin RR, Estey EH, et al: Targeting the leukemia microenvironment by CXCR4 inhibition overcomes resistance to kinase inhibitors and chemotherapy in AML. Blood 113: 6215-6224, 2009

22. Saba F, Soleimani M, Kaviani S, Abroun S, Sayyadipoor F, Behrouz S and Saki N: G-CSF induces up-regulation of CXCR4 expression in human hematopoietic stem cells by beta-adrenergic agonist. Hematology, 2014.

23. De La Luz Sierra M, Gasperini P, McCormick PJ, Zhu J and Tosato G: Transcription factor Gfi-1 induced by G-CSF is a negative regulator of CXCR4 in myeloid cells. Blood 110: 2276-2285, 2007.

24. Jadidi-Niaragh F, Yousefi M, Memarian A, Hojjat-Farsangi M, Khoshnoodi J, Razavi SM, Jeddi-Tehrani M and Shokri F: Increased frequency of $\mathrm{CD}^{+}$and $\mathrm{CD}^{+}$regulatory $\mathrm{T}$ cells in chronic lymphocytic leukemia: Association with disease progression. Cancer Invest 31: 121-131, 2013.

25. Filipazzi P, Huber V and Rivoltini L: Phenotype, function and clinical implications of myeloid-derived suppressor cells in cancer patients. Cancer Immunol Immunother 61: 255-263, 2012.

26. Raggi C, Mousa HS, Corrent M, Sica A and Invernizzi P: Cancer stem cells and tumor-associated macrophages: A roadmap for multitargeting strategies. Oncogene 35: 671-682, 2016.
27. Zou LH, Barnett B, Safah H, LaRussa VF, Evdemon-Hogan M, Mottram P, Wei S, David O, Curiel TJ and Zou W: Bone marrow is a reservoir for $\mathrm{CD} 4+\mathrm{CD} 25+$ regulatory $\mathrm{T}$ cells that traffic through CXCL12/CXCR4 signals. Cancer Res 64: 8451-8455, 2004.

28. Tabe Y and Konopleva M: Advances in understanding the leukaemia microenvironment. Br J Haematol 164: 767-778, 2014.

29. Kowanetz M, Wu X, Lee J, Tan M, Hagenbeek T, Qu X, Yu L, Ross J, Korsisaari N, Cao T, et al: Granulocyte-colony stimulating factor promotes lung metastasis through mobilization of Ly6G+Ly6C+ granulocytes. Proc Natl Acad Sci USA 107: 21248-21255, 2010.

30. Bayne LJ, Beatty GL, Jhala N, Clark CE, Rhim AD, Stanger BZ and Vonderheide RH: Tumor-derived granulocyte-macrophage colony-stimulating factor regulates myeloid inflammation and $\mathrm{T}$ cell immunity in pancreatic cancer. Cancer Cell 21: 822-835, 2012.

31. Kohanbash G, McKaveney K, Sakaki M, Ueda R, Mintz AH, Amankulor N, Fujita M, Ohlfest JR and Okada H: GM-CSF promotes the immunosuppressive activity of glioma-infiltrating myeloid cells through interleukin-4 receptor- $\alpha$. Cancer Res 73: 6413-6423, 2013.

32. Lin JP, Yang JS, Lu CC, Chiang JH, Wu CL, Lin JJ, Lin HL, Yang MD, Liu KC, Chiu TH and Chung JG: Rutin inhibits the proliferation of murine leukemia WEHI-3 cells in vivo and promotes immune response in vivo. Leuk Res 33: 823-828, 2009.

33. Wen YF, Yang JS, Kuo SC, Hwang CS, Chung JG, Wu HC, Huang WW, Jhan JH, Lin CM and Chen HJ: Investigation of anti-leukemia molecular mechanism of ITR-284, a carboxamide analog, in leukemia cells and its effects in WEHI-3 leukemia mice. Biochem Pharmacol 79: 389-398, 2010.

34. Mohan S, Abdul AB, Abdelwahab SI, Al-Zubairi AS, Aspollah Sukari M, Abdullah R, Taha MM, Beng NK and Isa NM: Typhonium flagelliforme inhibits the proliferation of murine leukemia WEHI-3 cells in vitro and induces apoptosis in vivo. Leuk Res 34: 1483-1492, 2010.

35. Mills KH: Regulatory T cells: Friend or foe in immunity to infection? Nat Rev Immunol 4: 841-855, 2004.

36. Wan YY and Flavell RA: TGF-beta and regulatory $\mathrm{T}$ cell in immunity and autoimmunity. J Clin Immunol 28: 647-659, 2008.

37. Romano A, Parrinello NL, Vetro C, Tibullo D, Giallongo C, La Cava P, Chiarenza A, Motta G, Caruso AL, Villari L, et al: The prognostic value of the myeloid-mediated immunosuppression marker Arginase-1 in classic Hodgkin lymphoma. Oncotarget 7: 67333-67346, 2016.

38. Munder M: Arginase: An emerging key player in the mammalian immune system. Br J Pharmacol 158: 638-651. 2009.

39. Meads MB, Hazlehurst LA and Dalton WS: The bone marrow microenvironment as a tumor sanctuary and contributor to drug resistance. Clin Cancer Res 14: 2519-2526, 2008

40. Tavor S and Petit I: Can inhibition of the SDF-1/CXCR4 axis eradicate acute leukemia? Semin Cancer Biol 20: 178-185, 2010

41. Ye M, Zhang H, Yang H, Koche R, Staber PB, Cusan M, Levantini E, Welner RS, Bach CS, Zhang J, et al: Hematopoietic differentiation is required for initiation of acute myeloid leukemia. Cell Stem Cell 17: 611-623, 2015.

42. Saito Y, Uchida N, Tanaka S, Suzuki N, Tomizawa-Murasawa M, Sone A, Najima Y, Takagi S, Aoki Y, Wake A, et al: Induction of cell cycle entry eliminates human leukemia stem cells in a mouse model of AML. Nat Biotechnol 28: 275-280, 2010

43. Konopleva MY and Jordan CT: Leukemia stem cells and microenvironment: Biology and therapeutic targeting. J Clin Oncol 29: 591-599, 2011.

44. Wei G, Ni W, Chiao JW, Cai Z, Huang H and Liu D: A meta-analysis of CA G(cytarabine, aclarubicin, G-CSF) regimen for the treatment of 1029 patients with acute myeloid leukemia and myelodysplastic syndrome. J Hematol Oncol 4: 46, 2011.

45. Liu L, Zhang Y, Jin Z, Zhang X, Zhao G, Si Y, Lin G, Ma A, Sun Y, Wang L and Wu D: Increasing the dose ofaclarubicin in low-dose cytarabine and aclarubicin in combination with granulocyte colony-stimulating factor (CAG regimen) can safely andeffectively treat relapsed or refractory acute myeloid leukemia. Int J Hematol 99: 603-608, 2014.

This work is licensed under a Creative Commons Attribution-NonCommercial-NoDerivatives 4.0 International (CC BY-NC-ND 4.0) License. 